\title{
Total thoracoscopic surgery for biatrial cardiac myxoma: a case report
}

\author{
Yipeng Tang", Jinghui Li", Feng Zhao, Tongyun Chen \\ Tianjin Chest Hospital, Tianjin University, Tianjin, China \\ \#These authors contributed equally to this work. \\ Correspondence to: Feng Zhao, MD; Tongyun Chen, MD. Tianjin Chest Hospital, Tianjin University, No. 261, Taierzhuangnan Road, Jinnan District, \\ Tianjin 300222, China. Email: zhaofeng7815@163.com; 35391185@qq.com.
}

\begin{abstract}
Myxoma is the most common type of benign cardiac tumor in adults. Myxoma can occur anywhere in the heart. The left atrium is the most frequent site of origin, specifically located on the left atrium side of the fossa oval in the atrial septum, followed by the right atrium, the right ventricle and left ventricle. But biatrial myxoma is extremely rare. Thoracoscopic resection of myxoma has become more common, but there are few reports on thoracoscopic surgery for biatrial myxoma. We present a case of a 72-year-old woman with biatrial myxoma, who presented with intermittent dyspnea for one week. Echocardiography revealed a medium echo in both the left and right atrium and was connected via the atrial septum. Computed tomography revealed a hypointense mass in both atria. Thoracoscopic resection successfully removed the tumors, and histological examination confirmed the diagnosis. Also, the patient was discharged six days after surgery. There was no evidence of tumor recurrence during the one-year followup period. Biatrial myxoma is rare. Surgical resection is the primary method for myxoma. Compared with the traditional medium thoracotomy, thoracoscopic surgery for myxoma has the following advantages: less trauma, keeping the integrity of the sternum, less bleeding, faster postoperative recovery, etc. Total thoracoscopic surgery for biatrial myxomas is effective and safe.
\end{abstract}

Keywords: Biatrial myxomas; thoracoscopic; case report

Submitted Sep 07, 2020. Accepted for publication Dec 02, 2020.

doi: 10.21037/atm-20-6993

View this article at: http://dx.doi.org/10.21037/atm-20-6993

\section{Introduction}

Cardiac myxoma is the most common primary benign cardiac tumor in adults (1). It is three times more common in women than in men. The average age of patients is 40-50 years (2). Children are very rare. Myxoma can occur anywhere in the heart. The left atrium is the most common (60-80\%), specifically located on the left atrium side of the fossa oval in the atrial septum, followed by the right atrium $(15-28 \%)$, the right ventricle $(8 \%)$ and left ventricle (3-4\%) (3). 90\% of cardiac myxomas are sporadic, and only $5-10 \%$ are family hereditary. Generally speaking, the symptoms of myxoma patients can be divided into the following categories: (I) abnormal hemodynamic manifestations: myxoma occur in the right atrium show symptoms of peripheral circulation ischemia, such as edema, hepatosplenomegaly, hepatic jugular veins Positive reflux sign, ascites, pleural effusion, etc.; myxoma occur in the left atrium, the symptoms of chest tightness, wheezing, coughing tenderness, sputum, hemoptysis, dyspnea, fatigue and other symptoms are the first manifestations. In severe cases, pulmonary hypertension may occur. When the myxoma is huge, it may completely block the atrioventricular valve orifice and cause syncope or sudden death. (II) Vascular embolism manifestations: due to the fragile texture of the tumor, it is easy to fall off. Vascular embolism is a common complication in patients with myxoma. Pulmonary embolism may occur in patients with tumor originating in the right atrium. Cerebral embolism, renal embolism, and limb arterial embolism 


\section{Page 2 of 6}

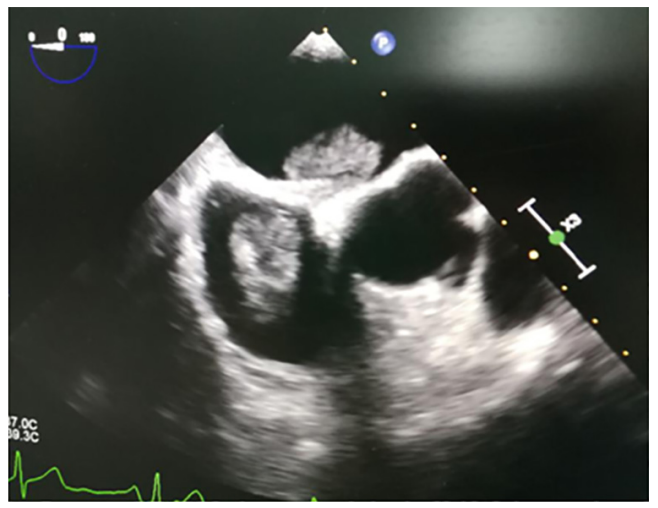

Figure 1 Transesophageal ultrasound shows the location of the bilateral atrial mass.

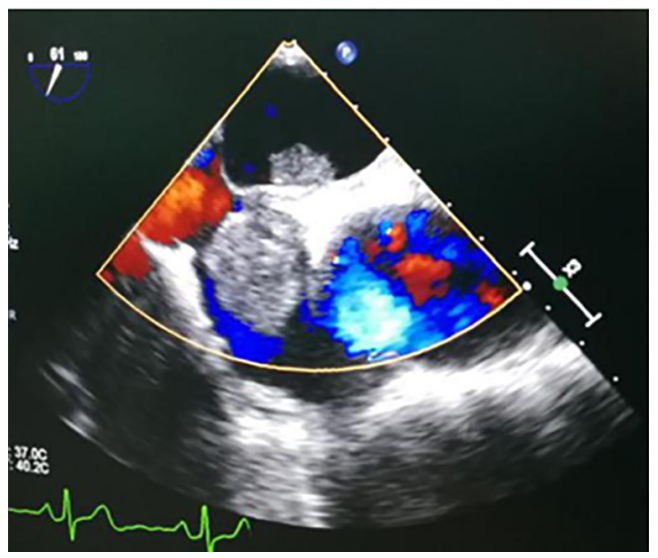

Figure 2 Transesophageal ultrasound showed that the mass did not affect the valve function.

may occur in patients with tumor originating in the left atrium. (III) Arrhythmia: the atrial conduction system may be abnormally due to the expansion of the atrial wall. Sometimes the tumor invades the conduction system, which can cause atrioventricular block. (IV) Systemic non-specific manifestations: in addition to the above main symptoms, patients may have unexplained low-grade fever, fatigue, joint pain, myalgia, accelerated erythrocyte sedimentation rate, anemia, thrombocytopenia and other systemic manifestations. It may be caused by the disorder of the immune system caused by the tumor.

Biatrial myxoma is rare to be found. Although thoracoscopic myxoma resection is standard, there are few reports on video-assisted biatrial myxoma resection. We present a case of biatrial myxoma resected by totally thoracoscopic surgery. The disease was discussed from
Tang et al. Total thoracoscopic surgery for bi-atrial cardiac myxoma

the aspects of preoperative diagnosis, formulation of the operation plan, and the key points of operation.

We present the following article in accordance with the CARE reporting checklist (available at http://dx.doi. org/10.21037/atm-20-6993).

\section{Case presentation}

A 72-year-old woman was admitted to our hospital complaining intermittent dyspnea for one week. She had no history of cardiac disease. On physical examination, the heart rate was regular at 87 beats $/ \mathrm{min}$, and the blood pressure was 132/67 mmHg. A grade II-III systolic murmur could be heard in the auscultation area of the tricuspid valve. The electrocardiogram shows normal sinus rhythm. A chest $\mathrm{X}$-ray revealed that the lung markings were thickened. Laboratory analyses were regular. Computed tomography revealed a hypointense mass in both atria. Transthoracic echocardiography reveals moderate echo masses in the left atrium and right atrium with a pedicle in the interatrial septum. The size of the left atrial mass is about $2.0 \mathrm{~cm} \times$ $1.6 \mathrm{~cm}$ with less range of motion, and the right atrial mass is about $3.6 \mathrm{~cm} \times 2.2 \mathrm{~cm}$ with a broader range of motion. Valve insufficiency was mild. Preoperative enhanced ultrasound of sulfur hexafluoride revealed insufficient blood supply in bilateral atria masses.

Surgery was performed 3 days after admission under a standard cardiopulmonary bypass, and total thoracoscopic surgery resected the myxoma. Transesophageal ultrasound revealed that pedicles of bilateral atrial masses attached to the atrial septum, and three-dimensional reconstruction was used to evaluate the shape, size, location, and adjacent areas of the tumor (Figures 1,2). A double-lumen endotracheal tube was inserted. During the operation, the single left lung ventilation was performed to support the patient. The patient was positioned in the left lateral position with the right hemithorax slightly elevated to a $30^{\circ}$ angle. The fourth intercostal incision $(3.5 \mathrm{~cm})$ was constructed into the right chest between the right midclavicular midline and the right axillary front line. The other two incisions $(1.5 \mathrm{~cm})$ were located in the anterior axillary line of the 4th intercostal space and the axillary midline of the 5 th intercostal space. Minimally invasive cardiopulmonary bypass was set up by catheterizing the right femoral artery, right femoral vein, and right internal jugular vein. We cut the right atrium and sutured the traction line. The tumor pedicle of the right atrium was located at the fossa ovalis. The tumor of the right atrium was carefully excluded from the pedicle with a 


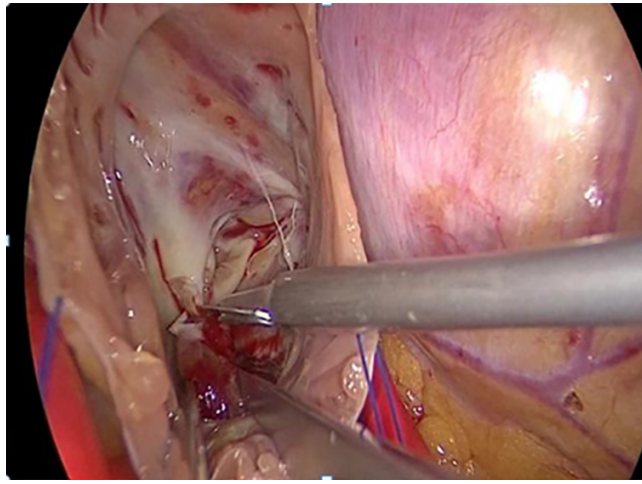

Figure 3 Right atrial myxoma under the endoscope.

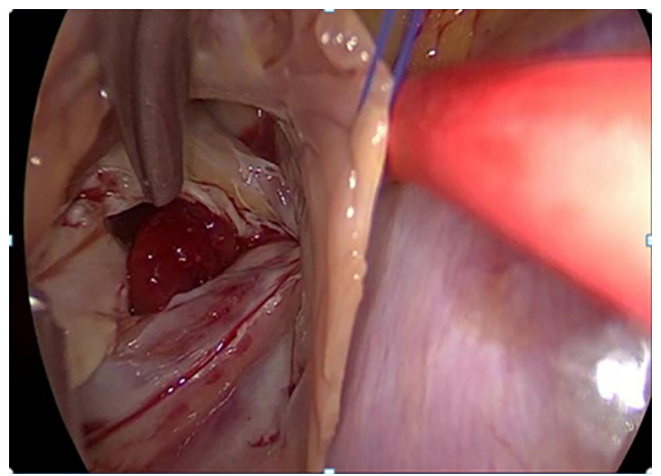

Figure 4 Left atrial myxoma under endoscope.

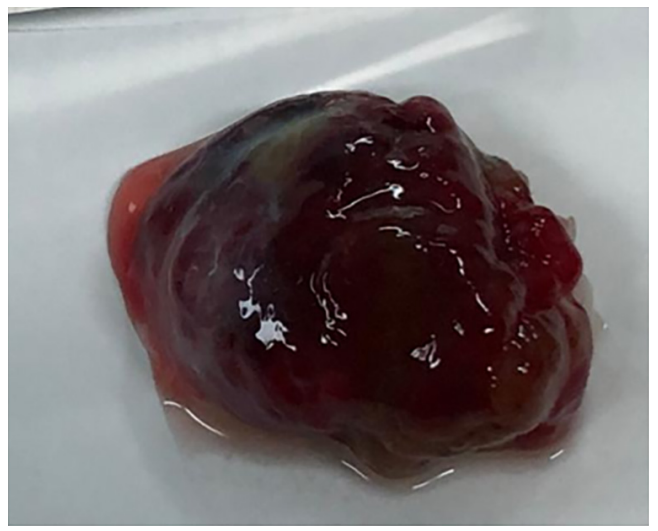

Figure 5 Right atrial myxoma specimen.

long-handled round knife and minimally invasive forceps, and the tumor $(3 \mathrm{~cm} \times 2 \mathrm{~cm}$, jelly-like, no evident capsule, fragile) was put into the endoscopic specimen collection bag and then taken out of the chest (Figure 3). After the atrial septum was opened, the left atrial tumor pedicle was located

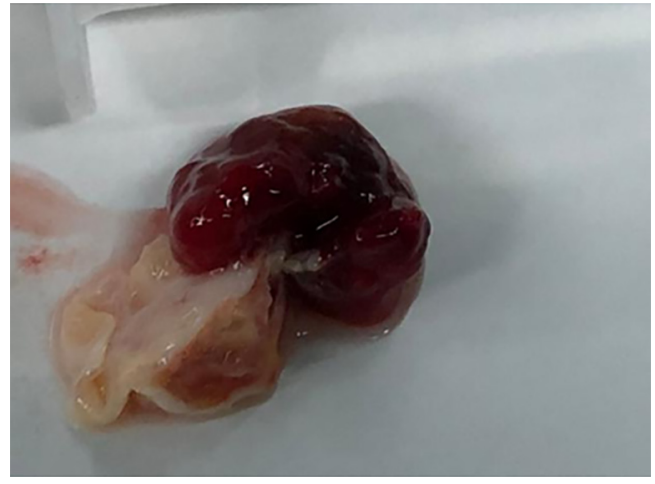

Figure 6 Left atrial myxoma specimen.

at the edge of the fossa ovalis, about $0.5 \mathrm{~cm}$, away from the contralateral point of attachment of the right atrial tumor pedicle, and the biatrial tumors were non-homologous.

The left atrial tumor $(2.4 \mathrm{~cm} \times 1.6 \mathrm{~cm}$, jelly-like, no apparent capsule, fragile) was carefully excised with a longhandled round knife, and the atrial septal tissue attached to the tumor pedicle of both atria was excised (Figure 4). After pruning the autologous pericardial patch fixed with glutaraldehyde, continuous suture repairs the atrial septal defect. The specimens of bilateral atrial masses are intact (Figures 5,6). Postoperative pathological analysis (Figure 7): left and right atrial masses are myxoma, immunohistochemistry: S100(-), Ki67(+<1\%). Special staining: PAS(-). The patient was discharged six days after surgery. There was no evidence of tumor recurrence during the one-year follow-up period.

All procedures performed in studies involving human participants were in accordance with the ethical standards of the institutional and/or national research committee(s) and with the Helsinki Declaration (as revised in 2013). Written informed consent was obtained from the patient.

\section{Discussion}

Cardiac myxomas have an incidence of $0.0017 \%$ among the general population (4). Sixty-five percent of myxomas occur in middle-aged women (5). Seventy-five percent of myxomas arise from the intra-atrial septum into the left atrium, $20 \%$ arise into the right atrium (6). Biatrial myxoma is relatively rare, accounting for about $2.5 \%$ (4). Seventyfive percent of biatrial myxomas are dumbbell-shaped or butterfly-shaped with two pedicles connected via the corresponding sides of the fossa ovalis (7). Biatrial myxoma with different pedicles is very rare. 

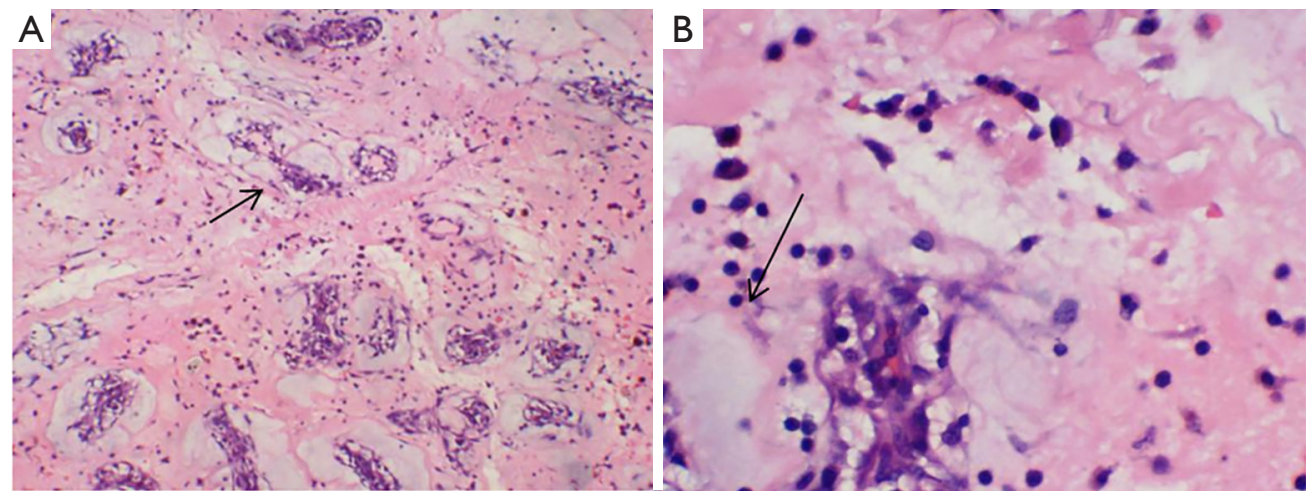

Figure 7 The postoperative pathological analysis left and right atrial masses were myxomas, immunohistochemistry: S100(-), Ki67(+<1\%). Special staining: Periodic Acid-Schiff stain (-). (Hematoxylin-eosin staining $\times 400)$.

The growth of cardiac myxoma in the cardiac cavity can cause blood flow obstruction, cardiac valve dysfunction, embolism, cardiac insufficiency, and even infection, which can be fatal (8). Biatrial myxoma may cause embolism of both left and right cardiac systems if tumor shedding occurs. Echocardiography is the simplest, safest, and reliable method for diagnosing cardiac myxoma. It can provide accurate judgment on the shape, mobility, attachment position of the tumor pedicle, the degree of damage to the atrioventricular valve, and the size of the atrioventricular. Surgical resection is the only effective treatment for cardiac myxoma. The world's first median thoracotomy left atrial myxoma resection was performed by Chitwood (9), which has been clinically proven to be safe and effective. In 1998, Ko reported for the first time 3 cases of left atrial myxoma removal through a minimally invasive small incision on the right side, filling the gap in completing the operation under a minimally invasive small incision (10). Subsequently, the technique of endoscopy has been greatly developed. Panos reported that thoracoscopic-assisted removal of left atrial myxoma was performed. All 10 patients successfully completed the operation. There was no termination of the operation or change of the operation style during the operation, and the postoperative recovery was good (11). Pineda (12) and Yu (13) et al. used the transatrial septum method to remove the myxoma of the left atrium. Minimally invasive surgery has cosmetic advantages and provides an excellent view of the inside of the left atrium through thoracoscopy. They also concluded that minimally invasive surgery for benign heart masses using a right-side approach is feasible and at least as safe as traditional total sternotomy.

Compared with the traditional medium thoracotomy, thoracoscopic surgery for myxoma has the following advantages: less trauma, keeping the integrity of the sternum, less bleeding, faster postoperative recovery, etc. In recent years, thoracoscopic surgery for myxoma has developed rapidly in China (14). At present, most of the reports in the literature are about the experience of endoscopic surgery for unilateral atrial myxoma, but there are few reports on thoracoscopic surgery for biatrial myxoma.

We summarize our experience in the treatment of this case of biatrial myxoma: (I) preoperative chest CT, transthoracic, and esophageal ultrasound were used to assess tumor size, tumor pedicle location, and the anatomical structure of the tumors. These assessments are essential for guiding the approach and the development of specific surgical protocols. (II) When establishing a minimally invasive cardiopulmonary bypass, we should pay attention that the right internal jugular vein drainage tube and the right femoral vein drainage tube cannot enter the right atrium to avoid the occurrence of a pulmonary embolism caused by the fragmentation of the right atrium tumor (15). (III) After preoperative evaluation, the excision of biatrial myxoma can be performed through a unilateral approach under totally thoracoscopic surgery. If the right atrial tumor is significantly larger than the left atrial tumor, we can first remove the right atrial tumor through the right atrial incision, and then remove the left atrial tumor from the atrial septal incision. However, if the tumor size of the left atrium is larger than the right atrium, we can remove the left atrial tumor through the left atrium incision, and then remove the right atrium tumor through the atrial septal incision. The long-term recurrence of myxoma can be prevented by taking out the tumor completely, complete resection of bilateral pedicle attachment tissue, and patch 
suture repair.

Patients with cardiac myxoma have good surgical treatments and generally have a good prognosis, but recurrence and malignant transformation may also occur. (I) Recurrence: the risk of recurrence of sporadic myxoma is about $3 \%$, and that of familial myxoma is $22 \%$. Recurrence is usually attributed to incomplete resection of the tumor, intracardiac implantation of the primary tumor, etc. (16). The risk of recurrence increases linearly in the first 4 years after resection of myxoma, and the risk of recurrence afterwards is very low (17). (II) Malignant transformation: the biological behavior of cardiac myxoma is benign, but a very small number of patients have malignant transformation (18). There are reports suggesting that patients with malignant transformation have genetic abnormalities. For such patients, surgical resection combined with chemotherapy and radiotherapy are given. Attention should be paid to follow-up after operation. For patients with tumor spread and cachexia, surgery should be carefully considered.

In summary, preoperative CT and preoperative ultrasound, especially transesophageal ultrasound, are essential for evaluating biatrial myxoma. According to the preoperative evaluation of tumor location and size, we can choose a different unilateral atrial approach in thoracoscopic surgery, which can improve the feasibility and safety of the operation.

\section{Limitation}

The patient's one-year postoperative review has been completed and the second year's postoperative review has not yet begun, so the follow-up time in this article is relatively short. This is also the shortcoming of this paper. We will continue to extend the follow-up time and pay attention to whether patients have adverse complications.

\section{Acknowledgments}

Funding: None.

\section{Footnote}

Reporting Checklist: The authors have completed the CARE reporting checklist. Available at http://dx.doi.org/10.21037/ atm-20-6993

Conflicts of Interest: All authors have completed the ICMJE uniform disclosure form (available at http://dx.doi. org/10.21037/atm-20-6993). The authors have no conflicts of interest to declare. All of authors not serve as a current Editorial Team member for this journal.

Ethical Statement: The authors are accountable for all aspects of the work in ensuring that questions related to the accuracy or integrity of any part of the work are appropriately investigated and resolved. All procedures performed in studies involving human participants were in accordance with the ethical standards of the institutional and/or national research committee(s) and with the Helsinki Declaration (as revised in 2013). Written informed consent was obtained from the patient.

Open Access Statement: This is an Open Access article distributed in accordance with the Creative Commons Attribution-NonCommercial-NoDerivs 4.0 International License (CC BY-NC-ND 4.0), which permits the noncommercial replication and distribution of the article with the strict proviso that no changes or edits are made and the original work is properly cited (including links to both the formal publication through the relevant DOI and the license). See: https://creativecommons.org/licenses/by-nc-nd/4.0/.

\section{References}

1. Keeling IM, Oberwalder P, Anelli-Monti M, et al. Cardiac myxomas: 24 years of experience in 49 patients. Eur J Cardiothorac Surg 2002;22:971-7.

2. Yoon DH, Roberts W. Sex distribution in cardiac myxomas. Am J Cardiol 2002;90:563-5.

3. Jain D, Maleszewski JJ, Halushka MK. Benign cardiac tumors and tumorlike conditions. Ann Diagn Pathol 2010;14:215-30.

4. Azari A, Moravvej Z, Chamanian S, et al. An unusual biatrial cardiac myxoma in a young patient. Korean J Thorac Cardiovasc Surg 2015;48:67-9.

5. Flint N, Siegel RJ, Bannykh S, et al. Bi-atrial cardiac myxoma with glandular differentiation: a case report with detailed radiologic-pathologic correlation. Eur Heart J Case Rep 2018;2:yty045.

6. González-Ferrer JJ, Carnero M, Labayru VL, et al. Left atrial myxoma prolapsing through the foramen ovale. Eur J Echocardiogr 2008;9:595-7.

7. Li Y, Li X, Wang X, et al. Biatrial myxoma floating like a butterfly: A case report and review of the literature. Medicine (Baltimore) 2018;97:e9558.

8. Liu D, Dong R. Clinical manifestation and surgical 
treatment analysis of five cases with biatrial myxoma. Int J Cardiol 2017;228:309-12.

9. Chitwood WR, Jr. Clarence Crafoord and the first successful resection of a cardiac myxoma. Ann Thorac Surg 1992;54:997-8.

10. Ko PJ, Chang CH, Lin PJ, et al. Video-assisted minimal access in excision of left atrial myxoma. Ann Thorac Surg 1998;66:1301-5.

11. Panos A, Myers PO. Video-assisted cardiac myxoma resection: basket technique for complete and safe removal from the heart. Ann Thorac Surg 2012;93:e109-10.

12. Pineda AM, Santana O, Zamora C, et al. Outcomes of a minimally invasive approach compared with median sternotomy for the excision of benign cardiac masses. Ann Thorac Surg 2011;91:1440-4.

13. Yu S, Xu X, Zhao B, et al. Totally thoracoscopic surgical resection of cardiac myxoma in 12 patients. Ann Thorac Surg 2010;90:674-6.

Cite this article as: Tang Y, Li J, Zhao F, Chen T. Total thoracoscopic surgery for biatrial cardiac myxoma: a case report. Ann Transl Med 2020;8(23):1608. doi: 10.21037/atm20-6993
14. Deng L, Zhang GW, Liu ZH, et al. Totally thoracoscopic surgery for atrial myxomas resection and atrial septal defect repair. Eur Rev Med Pharmacol Sci 2017;21:569-75.

15. Hanly J, de Buitleir M, Shaw K, et al. Bi-atrial myxomas presenting as recurrent pulmonary emboli in a girl. Postgrad Med J 1984;60:147-50.

16. Gosev I, Paic F, Duric Z, et al. Cardiac myxoma the great imitators: comprehensive histopathological and molecular approach. Int J Cardiol 2013;164:7-20.

17. Elbardissi AW, Dearani JA, Daly RC, et al. Survival after resection of primary cardiac tumors: a 48-year experience. Circulation 2008;118:S7-15.

18. Kakaty D, Grapow M, Huber B, et al. Long-term survival after extended resection of primary atrial myxosarcoma. J Surg Case Rep 2015;2015:rju146.

(English Language Editor: J. Chapnick) 